\title{
Tilted Pulse-Front Phase-matching in Three Dimensions: Overcoming The Cherenkov Angle Restrictions
}

\author{
Steven P. Jamison ${ }^{1}$, David A. Walsh ${ }^{2}$, Edward W. Snedden ${ }^{2}$ \\ ${ }^{1}$ Department of Physics, Lancaster University, Lancaster LA1 4YW, United Kingdom \\ ${ }^{2}$ Accelerator Science and Technology Centre, Science and Technology Facilities Council, Daresbury Laboratory, \\ Warrington, WA4 4AD, UK
}

\begin{abstract}
We consider the non-linear generation of $\mathbf{T H z}$ with tilted pulse-fronts in three dimensions and show that, contrary to the widely held expectations, coherent phase matching can be obtained for pulse-front tilt angles other the Cherenkov angle.
\end{abstract}

\section{INTRODUCTION}

$\mathrm{E}$ ffecient generation of high-field strength $\mathrm{THz}$ pulses are of interest for non-linear $\mathrm{THz}$ spectroscopy, and for application to $\mathrm{THz}$ driven particle acceleration. For many of the widely used non-linear materials with large non-linear coefficients phase-matching conditions preclude co-linear phase matching. For transversely narrow optical pump beams materials such as $\mathrm{LiNbO} 3$ give rise to $\mathrm{THz}$ emission in a Cherenkov cone around the drive laser. For transversely extended beams tilted pulse fronts are required to maintain efficient generation, and also have the desirable result of producing a planar front $\mathrm{THz}$ emission.

The generation of $\mathrm{THz}$ with tilted pulse fronts has been widely studied and exploited for over a decade [1-6]. It is generally accepted that to obtain the efficiency gains the pulse front tilt must match the Cherenkov angle of the material.

Recently, Walsh et al demonstrated a 'travelling source' $\mathrm{THz}$ generation concept for particle acceleration [7]. The generation scheme explicitly utilized a pulse-front tilt with tilt angle exceeding the Cherenkov angle, raising questions on the nature of the phase-matching efficiency. Here we show analytically, and also numerically through finite-difference time-domain modeling examples, that pulse-front tilt generation can meet the phase-matching condition whenever the tilt-angle exceeds the Cherenkov angle.

\section{RESULTS}

Previous derivations of Cherenkov angle pulse-front tilt phasematching have generally started from a two dimensional construction, requiring that the phase-fronts of the generated $\mathrm{THz}$ coherently reinforce in the 2-dimensional plane[1]. Similarly, published numerical modeling of the generation process has tended to assume a two-dimensional symmetry $[2,3]$. However for a 1-dimesional 'pencil-beam' excitation a 3 dimensional Cherenkov cone of emission is produced. By an explicitly three-dimensional construction of the Huygens-like emission throughout a finite, 3-dimensional volume it is possible to show analytically that constructive interference (i.e. phase-matching) can be obtained whenever the pulse-front tilt exceeds the Cherenkov angle. In Fig 1 we show results from a three dimensional FDTD modeling with pulse-front tilt equal to, and exceeding, the cherenkov angle. A planar wave $\mathrm{THz}$
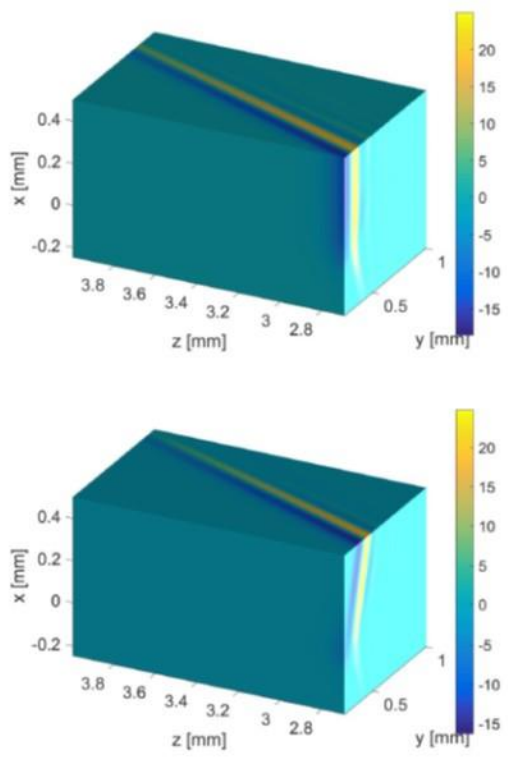

Fig. 1. THz generation of a planar front with differing pulse front tilt angle. Optical pulse propagation direction is in the z-direction. (a) with pulse-front tilt matching the Cherenkov angle of the material, with tilt in the $\mathrm{z}$-x plane. (b) with a pulse front tilt exceeding the Cherenkov angle. The pulse-front tilt has projection components in both the $\mathrm{zx}$ - and zy-planes. The angle projected into the $\mathrm{zx}$-plane is at the Cherenkov angle.

emission is obtained in both cases, and with equal efficiency and field strength.

\section{REFERENCES}

[1] Hebling, J., Almasi, G., Kozma, I.Z. Velocity matching by pulse front tilting for large area THz-pulse generation. Opt. Express. 10, 1161-1166 (2002)

[2] M.I. Baakunov, S.B. Bodrov, A.V. Maslov, M. Hangyo.Theory of terahertz generation in a slab of electro-optic material using an ultrashort laser pulse focused to a line. Phys Rev B 76, 085346 (2007)

[3] M.I. Bakunov, M.V. Tsarev, E.V. Mashkovich. Terahertz differencefrequency generation by tilted amplitude front excitation. Optics Express 20 $28573(2012)$

[4] Fulop, J. A., Palfalvi, L., Hoffmann, M. C. \& Hebling, J. Towards generation of mJ-level ultrashort THz pulses by optical rectification. IOpt. Express 19, 15090-15097 (2011).

[5] Hebling, J. Yeh, K-L, Hoffmann, M.C., Bartal, B., Nelson, K.A.

Generation of high-power terahertz pulses by tilted pulse front excitation and their application possibilities. J. Opt. Soc. Am B, 25, 6 (2008)

[6] Hirori, H., Doi, A., Blanchard, F. \& Tanaka, K. Single-cycle terahertz pulses with amplitudes exceeding $1 \mathrm{MV} / \mathrm{cm}$ generated by optical rectification in LiNbO3. Appl. Phys. Lett. 98, 091106 (2011).

[7]. D.A. Walsh, D.S. Lake, E.W. Snedden, M.J. Cliffe, D.M. Graham and S.P Jamison, "Demonstration of sub-luminal propagation of single-cycle terahertz pulses for particle acceleration," Nature Communications, vol. 8, pp. 421, 2017. 
